\title{
SIMBIOSIS BIOLOGÍA-EDUCACIÓN BAJO EL PRISMA DE LA CONTEXTUALIZACIÓN DE CONTENIDOS: EL CASO DEL MAR MENOR
}

\author{
María del Mar Rubio Leiva \\ Universidad de Murcia \\ Correspondencia: mdmruble@gmail.com \\ Para citar este artículo puede utilizar el siguiente formato: \\ María del Mar Rubio Leiva: "Simbiosis biología-educación bajo el prisma de la contextualización de \\ contenidos: el caso del Mar Menor", Revista Contribuciones a las Ciencias Sociales, (Especial \\ noviembre 2021, pp. 33-44). En línea: \\ https://doi.org/10.51896/CCS/LKCS7457
}

\section{RESUMEN}

El proceso de enseñanza-aprendizaje en Educación Secundaria Obligatoria (ESO) no tiene en cuenta la contextualización de contenidos en Biología y Geología, haciendo que los estudiantes no encuentren motivación por su estudio y presenten dificultades en el proceso de aprendizaje, provocando que éstos, a su vez, no adquieran actitudes en favor de la conservación y protección del medio natural. El presente estudio se enmarca en una clase de $1^{\circ}$ de ESO $(n=30)$ de un instituto localizado en el municipio de Murcia, con un nivel socioeconómico medio-alto. Se emplea una propuesta educativa, con una secuencia de actividades ( 6 sesiones de 15 actividades), en la que se pretende la contextualización de los contenidos relacionados con la dinámica de los ecosistemas. Para ello, se utiliza un ecosistema presente en la Región de Murcia para abordar los contenidos, concretamente, el Mar Menor, sirviendo de hilo conductor de dichas actividades. Con ello, los contenidos se encuentran contextualizados y se intenta promover la concienciación y sensibilización por la conservación y protección del medio ambiente. Para su implementación, se establece una selección de objetivos, relación con las competencias clave, análisis del libro de texto utilizado, diseño de las actividades y establecimiento del método de evaluación. Tras la implementación de la propuesta educativa, la mayoría de estudiantes muestra motivación por el estudio del temario, así como una mayor comprensión del mismo, indicando que la contextualización de los contenidos y uso variado de metodología, estrategias y recursos didácticos es positiva en el proceso de enseñanzaaprendizaje y promoción de actitudes proambientales.

Palabras clave: Biología, Educación, contextualización, ecosistemas, Educación Secundaria Obligatoria, Mar Menor. 


\section{CONTENTS: THE CASE OF THE MAR MENOR}

\section{ABSTRACT}

The teaching-learning process carried out in Secondary Education (ESO) does not take into account the contextualization of contents in Biology and Geology, triggering a lack of motivation in students when studying and having difficulties in the learning process, causing them, in turn, not to acquire attitudes in favor of the conservation and protection of the natural environment. The present study is framed in a class of 1 st of ESO $(n=30)$ of a high school located in the city of Murcia, with a medium-high socioeconomic level. An educational proposal is used, with a sequence of activities (6 sessions of 15 activities), in which the contextualization of the contents related to the dynamics of ecosystems is intended. For this, an ecosystem located in the Region of Murcia is used to address the contents, specifically, the Mar Menor ecosystem, serving as a common thread for these activities. With this, the contents are contextualized and an attempt is made to promote awareness and sensitization for the conservation and protection of the environment. For its implementation, a selection of objectives is established, related to the key competences, the students' textbook is analyzed, the activities are designed and the evaluation method is established. After the implementation of the educational proposal, most of the students show motivation for the study of the ecosystems and their dynamics, as well as a greater understanding of them, indicating that the contextualization of the contents and the varied use of methodology, strategies and didactic resources is positive in the teaching-learning process and promotion of pro-environmental attitudes.

Keywords: Biology, Education, contextualization, ecosystems, Secondary Education, Mar Menor.

\section{INTRODUCCIÓN}

Importancia de la contextualización de contenidos

Conocer las características de los ecosistemas y su dinámica, por parte del alumnado, presenta gran relevancia, no solo por la adquisición de conocimiento conceptual sobre éstos, sino también por la sensibilización ambiental que implica.

La sociedad, y concretamente los estudiantes de secundaria, presentan una escasa sensibilización ambiental y despreocupación ante el deterioro del patrimonio natural y el empobrecimiento de la diversidad biológica, en gran medida originados por el desconocimiento y una inadecuada educación ambiental (Santos-Ellakuria, 2019).

Actualmente, en España, el proceso de enseñanza-aprendizaje que se lleva a cabo en Educación Secundaria no tiene en cuenta la contextualización de los contenidos conceptuales tratados, así como las ideas previas y concepciones alternativas que el estudiantado suele presentar en relación a los mencionados contenidos (Hernández-Serrato y González-Reyes, 2021; Wyner y Blath, 2019). Por ello, el alumnado no encuentra motivación por su estudio, presentando dificultades varias en el proceso de aprendizaje y en la adquisición de actitudes en favor de la conservación y protección del medio natural (Rincón, 2011). 


\section{Condiciones de enseñanza-aprendizaje en el aula}

Los estudiantes de ESO mantienen una serie de ideas previas y concepciones alternativas relativas a la dinámica de los ecosistemas. Además, se encuentran dificultades específicas que pueden derivarse del aprendizaje de estos contenidos (García-Rodeja, Silva-García y Sesto-Varela, 2020). Con el conocimiento de estas dificultades e ideas previas, es posible la planificación de la enseñanza en torno a dichas ideas previas y concepciones alternativas, y desarrollar estrategias didácticas destinadas a solventar las posibles dificultades que acompañan a los contenidos.

Entre las ideas previas y concepciones alternativas que suelen presentar los estudiantes de primer curso de ESO, se encuentran las siguientes: consideran que las interacciones entre componentes bióticos se dan entre individuos y no entre poblaciones; creen que el suelo es el fin de la descomposición; no presentan visión sistémica del ecosistema; no diferencian la cadena trófica de la red trófica y consideran que las plantas elaboran alimento solo para el beneficio de animales y el ser humano, y no porque sea esencial para las propias plantas (Hernández-Serrato y GonzálezReyes, 2021). Asimismo, el alumnado no relaciona hongos y bacterias con el proceso de descomposición, no aplica la comprensión de las redes alimentarias en actividades cotidianas y piensa que el ecosistema solo se compone de seres vivos, no teniendo en cuenta los componentes abióticos ni estableciendo relación entre éstos y los componentes bióticos y en las relaciones tróficas solamente tienen en cuenta la relación depredador-presa (Wyner \& Blath, 2019).

En cuanto a las dificultades específicas que presenta el aprendizaje de estos contenidos, las más importantes son la concepción de los ecosistemas mediante un enfoque lineal y de causalidad simple, no teniendo en cuenta las interacciones que se llevan a cabo entre los distintos procesos; como consecuencia de ello, suelen entender el concepto de cadena trófica pero no el de red trófica (Rincón, 2011). Además, es preciso tener en cuenta la falta de aplicación de contenidos conceptuales a la vida real por parte de los estudiantes; es decir, su vida cotidiana, haciendo que los estudiantes contemplen dichos contenidos de forma descontextualizada (Wyner \& Blath, 2019). Por otra parte, generalmente, no se abordan los ecosistemas desde un punto de vista sistémico, por lo que el alumnado no suele entender el ecosistema como un sistema, presentando una visión limitada de su dinámica (Hernández-Serrato y González-Reyes, 2021).

A partir de las dificultades abordadas con anterioridad, es preciso considerar determinadas implicaciones didácticas. Así, la enseñanza que se plantea en el presente estudio, tiene en cuenta las ideas previas del alumnado, las dificultades de aprendizaje que las acompañan y las implicaciones o estrategias didácticas para tratar de solventarlas.

\section{Enmarcación del estudio}

Aunque los contenidos relacionados con los ecosistemas y la dinámica de éstos se abordan en dos cursos de Educación Secundaria Obligatoria (ESO) (Decreto 220/2015, de 2 de septiembre de 2015, por el que se establece el currículo de la Educación Secundaria Obligatoria en la 
Comunidad Autónoma de la Región de Murcia), y en Bachillerato (Decreto 221/2015, de 2 de septiembre de 2015, por el que se establece el currículo del Bachillerato en la Comunidad Autónoma de la Región de Murcia), es el primer curso de ESO, el curso académico en el que se debería profundizar en su estudio; pues para algunos estudiantes, supone la primera y última vez en estudiarlos. Por esta razón, el presente estudio queda enmarcado en el mencionado curso escolar (10 de ESO), a través de una propuesta educativa, con una secuencia de actividades, en la que se pretende la contextualización de los contenidos relacionados con la dinámica de los ecosistemas, acercándolos al alumnado para mejorar el proceso de enseñanza-aprendizaje, haciendo que éste sea significativo.

\section{METODOLOGÍA}

\section{Selección de objetivos, relación con las competencias clave y análisis del libro de texto}

Para la realización del presente estudio, se lleva a cabo, en primer lugar, una selección de los objetivos, tanto generales de etapa (ESO) (Real Decreto 1105/2014, de 26 de diciembre, por el que se establece el currículo básico de la Educación Secundaria Obligatoria y del Bachillerato), como específicos de la asignatura en la que se aborda (Biología y Geología) (Decreto 220/2015, de 2 de septiembre de 2015, por el que se establece el currículo de la Educación Secundaria Obligatoria en la Comunidad Autónoma de la Región de Murcia), quedando reflejados en la siguiente tabla (Tabla 1).

\section{Tabla 1}

Selección de objetivos, tanto generales de etapa (ESO) como específicos de la asignatura (Biología y Geología) que se encuentran relacionados con la propuesta educativa diseñada.

\section{OBJETIVOS RELACIONADOS CON LOS CONTENIDOS A ABORDAR}

\begin{tabular}{|c|c|}
\hline $\begin{array}{c}\text { Objetivos generales de etapa } \\
\text { (Educación Secundaria Obligatoria) }\end{array}$ & $\begin{array}{l}\text { Objetivos específicos de la asignatura } \\
\text { (Biología y Geología) }\end{array}$ \\
\hline $\begin{array}{l}\text { b) Desarrollar y consolidar hábitos de disciplina, } \\
\text { estudio y trabajo individual y en equipo como } \\
\text { condición necesaria para una realización eficaz de las } \\
\text { tareas del aprendizaje y como medio de desarrollo } \\
\text { personal. } \\
\text { f) Concebir el conocimiento científico como un saber } \\
\text { integrado, que se estructura en distintas disciplinas, } \\
\text { así como conocer y aplicar los métodos para } \\
\text { identificar los problemas en los diversos campos del } \\
\text { conocimiento y de la experiencia. } \\
\text { k) Conocer y aceptar el funcionamiento del propio } \\
\text { cuerpo y el de otros, respetar las diferencias, afianzar } \\
\text { hábitos de cuidado y salud corporales e incorporar la } \\
\text { educación física y práctica del deporte para favorecer } \\
\text { el desarrollo personal y social. Conocer y valorar la } \\
\text { dimensión humana de la sexualidad en toda su } \\
\text { diversidad. Valorar críticamente los hábitos sociales } \\
\text { relacionados con la salud, el consumo, el cuidado de } \\
\text { los seres vivos y el medio ambiente, contribuyendo a } \\
\text { su conservación y mejora. }\end{array}$ & $\begin{array}{l}\text { 1) Adquirir conocimientos y destrezas básicas que le } \\
\text { permitan adquirir una cultura científica. } \\
\text { 2) Asentar los conocimientos ya adquiridos. } \\
\text { 4) Valorar la importancia que la conservación del } \\
\text { medio ambiente tiene para todos los seres vivos. } \\
\text { 7) Entender y valorar la importancia de preservar el } \\
\text { medio ambiente por las repercusiones que tiene sobre } \\
\text { la salud. } \\
\text { 8) Aprender a ser responsable de sus decisiones } \\
\text { diarias y las consecuencias que las mismas tienen en } \\
\text { su salud y en el entorno que les rodea. } \\
\text { 10) Iniciarse en las grandes teorías que han permitido } \\
\text { el desarrollo más actual de esta ciencia. } \\
\text { 12) Afianzar la comprensión lectora, la expresión oral } \\
\text { y escrita, la argumentación en público y la } \\
\text { comunicación audiovisual. }\end{array}$ \\
\hline
\end{tabular}


Asimismo, se establece una relación con las siete competencias clave (Real Decreto 1105/2014, de 26 de diciembre, por el que se establece el currículo básico de la Educación Secundaria Obligatoria y del Bachillerato), atendiendo al grado de vinculación que presentan con la propuesta educativa (Tabla 2). Con ellas, se promueve el desarrollo del pensamiento científico a través de la adquisición de conocimientos, contrastación de ideas y aplicación de los descubrimientos al bienestar social, dotando a los estudiantes de la capacidad de identificar, plantear y resolver situaciones de la vida cotidiana.

La vinculación de la propuesta educativa con cada una de las competencias clave se establece mediante una escala de numeración, siendo 1 una nula vinculación y 3 la máxima vinculación posible. Con una puntuación de 2, aparecen las competencias clave que son abordadas durante la puesta en práctica de la propuesta didáctica, pero no de una forma profunda.

Tabla 2

Grado de vinculación de cada una de las competencias clave con la propuesta educativa diseñada.

\begin{tabular}{lc}
\hline \multicolumn{1}{c}{ Competencias clave } & $\begin{array}{c}\text { Vinculación con la propuesta } \\
\text { educativa }\end{array}$ \\
\hline Matemática y competencias básicas en Ciencia y Tecnología & 3 \\
Sociales y cívicas & 3 \\
Digital & 2 \\
Lingüística & 2 \\
Aprender a aprender & 2 \\
Sentido de iniciativa y espíritu emprendedor & 1 \\
Conciencia y expresiones culturales & 1 \\
\hline
\end{tabular}

Nota. Elaboración propia a partir del Real Decreto 1105/2014, de 26 de diciembre

Resulta evidente que la competencia matemática y las competencias básicas en Ciencia y Tecnología son abordadas de forma más profunda en comparación con el resto de competencias. Asimismo, la competencia social y cívica adquiere gran relevancia en la presente propuesta didáctica, promoviendo motivación de los estudiantes por el aprendizaje, generando curiosidad, haciendo que se sientan protagonistas del proceso y del resultado del mismo y consiguiendo al mismo tiempo una sensación de autoeficacia; con ello, se pretende la sensibilización por el medio natural. Además, aunque en menor medida, se contribuye al desarrollo de la competencia digital mediante la realización de actividades en las que el alumnado tiene que buscar información, empleando para ello la tecnología, como motores de búsqueda en un ordenador, utilización de software específico y herramientas tecnológicas determinadas. A su vez, se contribuye a la promoción de la competencia en comunicación lingüística mediante actividades en las que se lleve a cabo la acción comunicativa con otros interlocutores, a través de textos en múltiples modalidades, formatos y soportes.

Por otra parte, se lleva a cabo un análisis del libro de texto de la clase a la que se encuentra dirigida la propuesta educativa, con el fin de comprobar la presencia en él de las ideas previas del estudiantado, así como las dificultades de aprendizaje asociadas y diseño de estructuración de contenidos e imágenes. Para ello, se emplea el modelo de análisis establecido por MontañésBayonas y Jaén-García (2015). De dicho análisis se extrae que el libro de texto, no tiene en cuenta 
dichas ideas previas ni las dificultades de aprendizaje, limitándose a los contenidos recogidos en el Currículo Oficial, y la estructuración de contenidos e imágenes es mejorable, pudiendo generar dificultades en la comprensión de los mismos. Asimismo, las actividades no promueven el pensamiento científico ni la generación de actitudes proambientales.

En cuanto a la estructuración de contenidos en el libro de texto, ésta es compartimentada, apareciendo los contenidos de forma independiente, haciendo que el alumnado no adquiera una visión sistémica de los conceptos estudiados, generando errores conceptuales y dificultades en el proceso de aprendizaje. Por otra parte, las imágenes utilizadas son, en su mayoría, ilustraciones, por lo que el alumnado no percibe la realidad de forma directa; además, la colocación de las mismas es inadecuada, mostrando incoherencia con el texto.

Las actividades presentes en el libro de texto son, en su mayoría, de carácter cerrado y no tienen como objetivo la aplicación de conocimiento ni la generación de actitudes, requiriendo, en su mayoría, la reproducción de lo ya escrito en el propio libro de texto. Con ello, no se promueve en el alumnado la transmisión de valores y actitudes hacia la Ciencia y el medio ambiente.

\section{Establecimiento de objetivos de aprendizaje}

Además, se especifican los objetivos de aprendizaje que se pretenden cumplir con el desarrollo de la secuencia de actividades, diferenciando, a su vez, los objetivos conceptuales, procedimentales y actitudinales. Respecto a los objetivos conceptuales establecidos, éstos son: el reconocimiento de los tipos de relaciones que se pueden establecer dentro de un ecosistema, la comprensión de las diferencias entre biocenosis y biotopo, así como entre cadena y red trófica; saber cuándo un ecosistema se encuentra en equilibrio, reconocimiento de las acciones del ser humano sobre los ecosistemas y la situación problemática a la que se enfrentan en la actualidad los ecosistemas de la Región de Murcia.

En cuanto a los objetivos procedimentales, se establecen los siguientes: la proposición de soluciones frente al impacto ambiental que generan las acciones antrópicas sobre los ecosistemas, la interpretación y representación gráfica de cadenas y redes tróficas, la formulación de hipótesis ante un problema asociado a ecosistemas y la argumentación, con fundamentación científica, ante un debate en el que se planteen aspectos relacionados con los ecosistemas, los efectos de las acciones humanas en ellos y su actual deterioro.

Por su parte, los objetivos actitudinales incluidos en la presente propuesta educativa son: la valoración de la importancia de proteger el medio ambiente, adoptando medidas críticas ante su deterioro, la identificación de acciones perjudiciales y beneficiosas para la conservación y protección del medio natural y la búsqueda de información sobre ecosistemas con espíritu crítico.

\section{Propuesta inicial de contenidos}

Asimismo, en la planificación de la presente propuesta didáctica es necesario señalar cuáles 
son los contenidos que se abordan durante la puesta en práctica de la misma. Dichos contenidos se clasifican en tres modalidades, de carácter conceptual, procedimental y actitudinal (Tabla 3).

Tabla 3

Relación de contenidos conceptuales, procedimentales y actitudinales que se abordan en la propuesta educativa.

\begin{tabular}{|c|c|}
\hline Contenidos conceptuales & $\begin{array}{l}\text { Hábitat, niveles tróficos, productores, consumidores, descomponedores, niveles } \\
\text { tróficos, red trófica, relaciones intraespecíficas, relaciones interespecíficas, } \\
\text { asociaciones, equilibrio, factor limitante, factores desencadenantes de } \\
\text { desequilibrios, gestión ambiental. }\end{array}$ \\
\hline $\begin{array}{l}\text { Contenidos } \\
\text { procedimentales }\end{array}$ & $\begin{array}{l}\text { Formulación de hipótesis, determinación de factores que inciden en el } \\
\text { comportamiento de un fenómeno, utilización de diversas fuentes de información, } \\
\text { elaboración de argumentos con fundamentación científica para la participación } \\
\text { en debates, búsqueda de información avanzada, representación gráfica de } \\
\text { formas de alimentación de los seres vivos a través de cadenas tróficas y redes } \\
\text { tróficas, elaboración de un esquema resumen con los conceptos más } \\
\text { importantes relacionados con la dinámica de los ecosistemas. }\end{array}$ \\
\hline Contenidos actitudinales & $\begin{array}{l}\text { Valorar la importancia de la conservación de los ecosistemas, sensibilización por } \\
\text { el medio ambiente, concienciación de la importancia que tiene el entorno natural } \\
\text { para el desarrollo de la vida humana, valoración crítica de los efectos } \\
\text { degradantes del ser humano; promover la defensa y conservación del medio } \\
\text { ambiente mediante la participación activa en el cuidado del mismo con } \\
\text { argumentos fundamentados, ante actividades humanas responsables de } \\
\text { contaminación y degradación, así como la consideración y respeto por opiniones } \\
\text { y aportaciones de otros, reconociendo la importancia del trabajo en equipo para } \\
\text { la construcción de conocimiento. }\end{array}$ \\
\hline
\end{tabular}

Nota. Elaboración propia.

\section{Diseño de la secuencia de actividades}

En cuanto al diseño de la secuencia de actividades, se emplea un ecosistema presente en la Región de Murcia, para abordar los distintos contenidos en la secuencia de actividades. Concretamente, se emplea el Mar Menor, sirviendo de hilo conductor de la mencionada secuencia, contextualizando así todos los contenidos en algo cercano para los estudiantes, de forma que tengan sentido para ellos, despertando su curiosidad hacia las Ciencias y haciendo que se encuentren motivados durante el proceso de aprendizaje. Asimismo, se les hace partícipes del proceso, implicándolos en la generación de soluciones ante la problemática que azota al Mar Menor en la actualidad. De esta forma, no solo se encuentran los contenidos contextualizados, sino que, a su vez, se promueve la concienciación y sensibilización por la conservación y protección del medio ambiente.

La secuencia consta de quince actividades, en las que se emplea variedad de metodologías, estrategias y recursos didácticos. Estas quince actividades abarcan un total de seis sesiones en las que se tienen en cuenta las cuatro etapas que conforman el proceso de enseñanza-aprendizaje (Angulo y García-Rovira, 1997), siendo éstas la etapa de exploración, introducción del conocimiento, estructuración y aplicación.

La primera etapa, la de exploración, en la que se parte de situaciones reales, concretas y simples en las que se presentan los conceptos que se quieren enseñar desde diversos puntos de vista para que los estudiantes sepan cuál será el objeto del aprendizaje y su utilidad, y para 
reconocer cuáles son las concepciones de los estudiantes sobre el tema.

La segunda etapa es la de introducción del conocimiento, en la que se plantean situaciones progresivamente más abstractas, empezando por las más intuitivas y manipulativas, que faciliten a los estudiantes la construcción del conocimiento.

La tercera etapa que conforma el proceso de enseñanza-aprendizaje es la de estructuración, cuyo objetivo principal es que los estudiantes sean capaces de organizar y jerarquizar sus nuevos conocimientos, familiarizándose con el contenido introducido y reconociendo el modelo construido. En esta etapa de la secuencia se incluyen actividades de síntesis y estructuración lógica.

La cuarta etapa que forma parte del proceso de enseñanza-aprendizaje es la de aplicación, en la que se lleva a cabo la aplicación del concepto a situaciones reales concretas para interpretar la realidad, saber utilizar el nuevo aprendizaje y reconocer su utilidad.

Asimismo, en el diseño de las actividades, se tiene en cuenta la situación sanitaria por pandemia de COVID-19 presente en España y, concretamente, en la Región de Murcia, en el momento de la puesta en práctica de la propuesta educativa. Por ello, las actividades diseñadas pretenden evitar el contagio vírico, a partir del establecimiento de distancia de seguridad entre estudiantes, proyección de las actividades para evitar el contacto de material entre personas distintas, y realización de actividades de forma individual para evitar agrupamientos en los que se tengan que romper la distancia sanitaria.

\section{Método de evaluación}

Para comprobar la eficacia de la secuencia diseñada, se llevan a cabo dos procesos distintos de evaluación. El primero de ellos, relacionado con la evaluación del aprendizaje que han adquirido los estudiantes, a través de rúbricas de evaluación de algunas de las actividades incluidas en dicha secuencia; y el segundo, relacionado con la evaluación del proceso de enseñanza, a través de fichas de observación y opiniones de los estudiantes.

\section{Muestra}

Para la realización del presente estudio, se emplea una muestra de 30 estudiantes que se encuentran cursando $1^{\circ}$ de ESO. Dicho alumnado estudia en un Instituto de Educación Secundaria (IES) público, localizado en el municipio de Murcia (Región de Murcia, España), concretamente, en un área con un nivel socio-económico medio-alto.

En total, el centro escolar cuenta con 2550 estudiantes, distribuidos entre los distintos niveles y modalidades de enseñanza que se imparten en el centro y 140 docentes de distintas especialidades que imparten docencia en él.

La muestra está formada por 18 alumnas y 12 alumnos, todos ellos cursando el primer curso 
de ESO por primera vez, en el momento de la realización del estudio. La clase en la que se pretende implementar la propuesta didáctica es de carácter ordinario. Estos estudiantes mantienen una buena relación con el profesorado en general, y con el docente que les imparte la asignatura de Biología y Geología (materia objeto de estudio del presente estudio); además, entre ellos la relación es buena y muestran respeto y capacidad de trabajo en equipo, manteniendo un buen clima de aula.

\section{Atención a la diversidad}

Con el fin de garantizar que la totalidad del alumnado acceda a una educación de calidad en igualdad de oportunidades, resulta de gran importancia la atención a la diversidad de éste (Real Decreto 1105/2014, de 26 de diciembre).

De forma general, se plantean dos acciones, en primer lugar, la planificación de actividades destinadas al aprendizaje universal, estando adaptadas al ritmo individual de aprendizaje de cada estudiante, y en caso de que las circunstancias lo requieran, es posible aplicar los denominados planes de trabajo individualizados (PTI) no significativos, cuando los estudiantes no pertenezcan al grupo de estudiantes con necesidades específicas de apoyo educativo (ACNEAE). Por otra parte, es posible el desarrollo del PTI para el alumnado que pertenezca al mencionado grupo, estando dicha acción realizada en coordinación con el tutor de cada estudiante, el equipo docente y el Departamento de Orientación del centro escolar. Durante el desarrollo de dicho plan, es posible que se tenga que llevar a cabo una adaptación curricular individualizada $(A C l)$, en la cual, se deben especificar los estándares de aprendizaje trabajados con el propio estudiante.

En la presente propuesta educativa, se tiene en cuenta la atención a la diversidad de forma ordinaria, se emplea una amplia variedad de estrategias metodológicas y recursos didácticos, desarrollando actividades tanto de forma individual como en grupos de distintos integrantes. En la clase objeto de estudio, ninguno de los estudiantes es ACNEAE, y por ello, no se presenta necesario el empleo de medidas específicas de atención a la diversidad del alumnado.

\section{RESULTADOS Y DISCUSIÓN}

Tras la implementación en el aula de la propuesta educativa diseñada, se establece que la mayoría de los estudiantes responden las cuestiones con una calidad media-alta, relacionando en ocasiones los contenidos con otros vistos con anterioridad. La mayor parte de los estudiantes muestra un dominio de los términos adecuado, comprendiendo la diferencia entre red trófica y cadena trófica, pudiendo representar con solvencia ambos tipos de esquemas $(70 \%$ de los estudiantes).

Por otra parte, en general, la participación y el nivel de atención del alumnado son bastante altos, mostrando continuamente interés por las actividades y motivación por su resolución a lo largo de toda la secuencia.

Asimismo, ante distintos problemas ambientales, los estudiantes son capaces, en su 
mayoría, de proponer soluciones adecuadas, pero no con una calidad tan alta como la esperada y sin una argumentación sólida. Además, un $40 \%$ de estudiantes sabe reconocer el nivel trófico al que pertenecen los seres vivos en una red trófica, y la mayoría (90\%) reconoce la importancia que cada uno de los niveles tróficos tiene en una red trófica, identificando, por tanto, los posibles desencadenantes en caso de que alguno de dichos niveles tróficos quede afectado.

Un 55\% de los estudiantes sabe identificar la introducción de especies exóticas invasoras como una acción perjudicial para el ecosistema, y el 30\% de ellos reconoce el estado de equilibrio que se puede desencadenar por la introducción de este tipo de especies. Asimismo, el 90\% del alumnado busca información relacionada con ecosistemas, utilizando diversos motores de búsqueda, haciéndolo de forma eficaz y empleando el espíritu crítico.

En cuanto al reconocimiento de los efectos que las acciones antrópicas generan sobre los ecosistemas, el $90 \%$ del alumnado contesta positivamente tras la aplicación de la propuesta educativa. Asimismo, el $50 \%$ de los estudiantes formula hipótesis adecuadas en cuanto a las posibles consecuencias y soluciones que se puedan establecer ante una situación de desequilibrio de un ecosistema; no obstante, la calidad de la fundamentación de los argumentos propuestos no es satisfactoria.

Por otra parte, la mayoría de estudiantes, concretamente, el $80 \%$, comprende las relaciones que se establecen dentro de un ecosistema, siendo capaces de reconocer dichas relaciones ante casos prácticos propuestos. Además, el 75\% del alumnado es capaz de discernir la diferencia entre biotopo y biocenosis.

Con ello, la mayoría de los objetivos de aprendizaje establecidos en el diseño metodológico del estudio, se cumplen con la puesta en práctica de la propuesta educativa.

\section{Limitaciones de la propuesta}

El presente estudio consta de una serie de limitaciones, siendo algunas de ellas fruto de la propia naturaleza de la práctica educativa. Uno de los factores limitantes es el tamaño de la muestra (30 estudiantes), pues no es elevado, y por ello, puede suponer una limitación a la hora de establecer conclusiones al respecto. Asimismo, se ha de tener en cuenta que se trata de personas, y tanto el estado físico como mental del alumnado, puede repercutir en los resultados obtenidos y, consecuentemente, en las cuestiones investigadas.

Por otra parte, la falta de grupo control limita las conclusiones en el estudio, dificultando la interpretación de los datos obtenidos, resultando complicado saber si otros factores que tienen lugar al mismo tiempo que la puesta en práctica de la presente propuesta, guardan relación causal con lo estudiado.

Además, otro factor limitante a tener en cuenta es la situación sanitaria por la pandemia de COVID-19 en el momento de la puesta en práctica de la propuesta didáctica. Aunque el diseño de las 
actividades se lleva a cabo teniendo en cuenta este elemento limitante, durante la puesta en práctica es posible que se tenga que llevar a cabo alguna modificación; por ello, se ha intentado diseñar la secuencia de actividades de manera flexible para que no resulte compleja la modificación del mencionado diseño y para que la temporalización establecida en su inicio pueda ser llevada a la práctica de forma flexible en el tiempo, pudiendo requerir una mayor duración de la estimada a su comienzo.

\section{CONCLUSIONES}

Las valoraciones dadas por el estudiantado, así como el desarrollo y resultados de las actividades propuestas, indican que la mayoría de los estudiantes comprenden los contenidos y se promueve la sensibilización ambiental en ellos. A través del uso variado de estrategias didácticas y contextualización de contenidos, se promueve que el estudiantado se formule preguntas que le guíen hacia la comprensión de contenidos y, al mismo tiempo, de los fenómenos que le rodean; fomentando, a su vez, la reflexión, razonando y sabiendo comunicar lo aprendido.

Ante lo anteriormente expuesto, se establece que el desarrollo de la propuesta educativa en clase ha sido exitoso, tanto desde el punto de vista del alumnado como de la enseñanza por parte del docente y diseño de la propuesta educativa; resultando, por tanto, positiva la contextualización de los contenidos y uso variado de metodología, estrategias y recursos didácticos.

\section{REFERENCIAS}

Angulo-Delgado, F. y García-Rovira, M. P. (1997). Aprender a enseñar ciencias: una propuesta basada en la autorregulación. Revista Electrónica Interuniversitaria de Formación del Profesorado, 1(0). http://revistas.um.es/reifop/index

Decreto 220/2015, de 2 de septiembre de 2015, por el que se establece el currículo de la Educación Secundaria Obligatoria de la Comunidad Autónoma de la Región de Murcia. BORM, 203, 30729-31593.

Decreto 221/2015, de 2 de septiembre de 2015, por el que se establece el currículo del Bachillerato en la Comunidad Autónoma de la Región de Murcia. BORM, 203, 31594-32545.

Hernández-Serrato, C. y González-Reyes, R. (2021). Aprendizaje del concepto de red trófica. Un análisis desde el pensamiento lineal y sistémico. Revista Boletín Redipe, 10(1), 272-289. http://doi.org/10.36260/rbr.v10i1.1179

Montañés-Bayonas, S. y Jaén-García, M. (2015). ¿Qué características presentan los contenidos relacionados con las problemáticas ambientales propuestos en los libros de texto de $3^{\circ}$ de la eso?*. Revista Eureka sobre Enseñanza y Divulgación de las Ciencias, 12(1), 130-148. https://revistas.uca.es/index.php/eureka/index

Real Decreto 1105/2014, de 26 de diciembre, por el que se establece el currículo básico de la Educación Secundaria Obligatoria y del Bachillerato. BOE, 3.

Rincón, M. (2011). Concepciones de los estudiantes de educación básica sobre el ecosistema. Una revisión documental. Revista Bio-grafía: Escritor sobre la biología y su enseñanza, 4(7), 7793. http://doi.org/10.17227/20271034.7biografia77.93 
Santos-Ellakuria, I. (2019). Fundamentos para el aprendizaje significativo de la biodiversidad basados en el constructivismo y las metodologías activas. Revista de Innovación y Buenas Prácticas Docentes, 8(2), 90-101. http://doi.org/10.21071/ripadoc.v8i2.12170

Wyner, Y. \& Blath, E. (2019). Connecting ecology to daily life: how students and teachers relate food webs to the food they eat. Journal of Biological Education, 53(2), 128-149. http://doi.org/10.1080/00219266.2018.1447005 